\title{
Identification of factors that function in Drosophila salivary gland cell death during development using proteomics
}

\author{
CK McPhee ${ }^{1,2,3}$, BM Balgley ${ }^{4}$, C Nelson ${ }^{1}$, JH Hill ${ }^{1,2,5}$, Y Batlevi ${ }^{1}$, X Fang $^{6}$, CS Lee ${ }^{6}$ and EH Baehrecke ${ }^{*, 1}$
}

Proteasome inhibitors induce cell death and are used in cancer therapy, but little is known about the relationship between proteasome impairment and cell death under normal physiological conditions. Here, we investigate the relationship between proteasome function and larval salivary gland cell death during development in Drosophila. Drosophila larval salivary gland cells undergo synchronized programmed cell death requiring both caspases and autophagy (Atg) genes during development. Here, we show that ubiquitin proteasome system (UPS) function is reduced during normal salivary gland cell death, and that ectopic proteasome impairment in salivary gland cells leads to early DNA fragmentation and salivary gland condensation in vivo. Shotgun proteomic analyses of purified dying salivary glands identified the UPS as the top category of proteins enriched, suggesting a possible compensatory induction of these factors to maintain proteolysis during cell death. We compared the proteome following ectopic proteasome impairment to the proteome during developmental cell death in salivary gland cells. Proteins that were enriched in both populations of cells were screened for their function in salivary gland degradation using RNAi knockdown. We identified several factors, including trol, a novel gene CG11880, and the cop9 signalsome component cop9 signalsome 6, as required for Drosophila larval salivary gland degradation.

Cell Death and Differentiation (2013) 20, 218-225; doi:10.1038/cdd.2012.110; published online 31 August 2012

Programmed cell death is critical to multi-cellular animal development and homeostasis, and misregulation of cell death can lead to disorders including autoimmunity and cancer. ${ }^{1-3}$ Three prominent morphological forms of programmed cell death occur during development, including apoptosis, programmed necrosis, and autophagic cell death. ${ }^{4,5}$ Autophagic cell death is characterized by the induction of autophagy in dying cells that are degraded and removed without known phagocytic uptake. ${ }^{4}$ Although our understanding of autophagic cell death has recently increased, how autophagy and other catabolic processes promote cell death and degradation remains poorly understood.

Cells use two general catabolic processes to degrade and recycle cellular components, the ubiquitin proteasome system (UPS) and autophagy. During autophagy, cytoplasmic contents are sequestered by the autophagosome and delivered to the lysosome for degradation. Proteins to be degraded by the UPS are polyubiquitinated and targeted to the proteasome, an ATP-dependent 26S multisubunit protease complex. ${ }^{6}$ Whereas the UPS is used to degrade short-lived proteins, autophagy is generally thought to degrade long-lived proteins. Although the fundamental processes of autophagy and the UPS are understood, less is known about the function of these processes during programmed cell death.
The UPS has a key role in multiple cellular processes, including cell cycle regulation ${ }^{7}$ and antigen processing. ${ }^{8}$ Studies have also suggested a role for the UPS in coordinating cell death. ${ }^{9}$ Studies in cell lines suggest that caspase-dependent proteasome impairment may facilitate cell death. ${ }^{10,11}$ Furthermore, changes in ubiquitination and proteasome composition occur during hawkmoth development in dying intersegmental muscle cells. $^{12-15}$ Interestingly, these cells die with autophagic cell death morphology, suggesting a possible role for the UPS in autophagic cell death, but this has not been tested empirically.

Several studies suggest that ectopic proteasome impairment induces cell death in cell lines, ${ }^{16,17}$ and drug-induced proteasome impairment in tumor cells results in caspase activation and cell death. ${ }^{18}$ In addition, proteasome inhibitors are used in cancer therapies. ${ }^{19}$ The reversible proteasome inhibitor Bortezomib (Velcade, Millennium Parmaceuticals, Cambridge, MA, USA) is used to treat myeloma and lymphoma. ${ }^{20}$ Evidence in cell lines suggests that proteasome inhibitors lead to cell death by downregulating NF- $\mathrm{B}$ signaling ${ }^{21,22}$ and/or by inducing endoplasmic-reticulum stress. ${ }^{23,24}$ However, little is known about the effects of proteasome inhibitors on cellular processes, and how proteasome inhibition leads to cell death

\footnotetext{
${ }^{1}$ Department of Cancer Biology, University of Massachusetts Medical School, Worcester, MA 01605, USA; ${ }^{2}$ Department of Cell Biology and Molecular Genetics, University of Maryland, College Park, MD 20742, USA; ${ }^{3}$ Department of Molecular and Cellular Biology, Harvard University, Cambridge, MA 02138, USA; ${ }^{4}$ Bioproximity LLC, Springfield, VA 22150, USA; ' 5 Laboratory of Molecular Genetics, National Heart, Lung, and Blood Institute, National Institutes of Health, Bethesda, MD 20892, USA and ${ }^{6}$ Department of Chemistry and Biochemistry, University of Maryland, College Park, MD 20742, USA

*Corresponding author: EH Baehrecke, Department of Cancer Biology, University of Massachusetts Medical School, Lazare Research Building, Room 423, Worcester, MA 01605, USA. Tel: 508856 6733; Fax: 508856 1310; E-mail: Eric.Baehrecke@umassmed.edu

Keywords: autophagy; programmed cell death; ubiquitin proteasome system; proteomics; cop9 signalsome; trol/perlecan

Abbreviations: UPS, ubiquitin proteasome system; MS, mass spectrometry; CSN6, cop9 signalsome 6

Received 9.1.12; revised 30.6.12; accepted 10.7.12; Edited by RA Knight; published online 31.8.12
} 
has not been investigated under physiological conditions in vivo.

Larval salivary gland cells undergo synchronized programmed cell death requiring both caspases and autophagy (Atg) genes during Drosophila development. ${ }^{25}$ In wild-type Drosophila, the onset of salivary gland cell death occurs $12 \mathrm{~h}$ after puparium formation (APF), and DNA fragmentation and caspase activity can be detected at this time. Between 13 and $14 \mathrm{~h} \mathrm{APF}$, autophagy is induced and salivary glands become smaller in size, or condensed. By $16 \mathrm{~h}$ APF, wild-type salivary glands are completely degraded.

Recent developments in high-throughput mass spectrometry (MS)-based proteomic technologies prompted us to conduct a new proteomic analysis of dying salivary glands in Drosophila. We found that among the groups of proteins identified, UPS components were the top category enriched in wild-type dying salivary glands, suggesting a possible role for the UPS in larval salivary gland cell death. We monitored UPS function in salivary gland cells and found that flux through the UPS is reduced during wild-type salivary gland cell death. Significantly, premature proteasome impairment using an inducible genetic proteasome mutant leads to the onset of early salivary gland condensation (an overall reduction in salivary gland size) and DNA fragmentation, as evidenced by TUNEL staining. Surprisingly, we find that the expression of the caspase inhibitor p35 during proteasome impairment does not suppress early salivary gland condensation. This suggests the involvement of caspase-independent factors in the cellular response to proteasome impairment leading to ectopic salivary gland condensation. To identify factors that function in the response to proteasome impairment during autophagic cell death, we conducted proteomic analyses of salivary glands following ectopic proteasome impairment. We used a genetic approach to screen the top candidates identified in our proteomics data sets for defects in salivary gland degradation. Here, we show that trol, CG11880, a gene of unknown function, and the cop9 signalsome component csn6, are required for salivary gland cell degradation.

\section{Results}

UPS components are enriched in dying Drosophila larval salivary glands. During Drosophila development, autophagic programmed cell death of larval salivary glands is triggered by a rise in the steroid hormone 20-hydroxyecdysone (ecdysone) $12 \mathrm{~h}$ after APF, and requires both caspases and autophagy. ${ }^{25-27}$ In wild-type animals, salivary glands begin to die $12 \mathrm{~h}$ APF, and DNA degradation and caspase activation can be detected at this time in salivary gland cells. By 13-14h APF, autophagy is induced and salivary glands become smaller in size, or condensed. Thereafter, salivary glands are rapidly degraded and are absent by $16 \mathrm{~h}$ APF.

To identify new proteins required for larval salivary gland cell degradation, we isolated proteins from purified larval salivary glands before (6h APF), during (12 h APF), and after $(13.5 \mathrm{~h} \mathrm{APF})$ the onset of salivary gland cell death (Supplementary Table S1). In the 6-h samples, we identified an average of 12487 distinct peptides that mapped to 4157 distinct proteins with $>95 \%$ confidence (Supplementary
Table S2). Similarly, in the 12-h samples, we identified an average of 11530 distinct peptides that mapped to 3566 distinct proteins, and in the 13.5-h samples, we identified an average of 13012 distinct peptides that mapped to an average of 4184 distinct proteins with $>95 \%$ confidence (Supplementary Table S2).

Using this proteomic approach, proteins previously shown to be involved in salivary gland programmed cell death were identified, including the ecdysone receptor component Usp, the ecdysone response protein Eip93F (E93), the nuclear receptor competence factor $\beta F t z-f 1$, the Drosophila caspase protease Ice (Drlce), and several autophagy proteins (Supplementary Table S3). These data are consistent with published DNA microarray and proteomics studies, ${ }^{28,29}$ as well as genetic studies showing that these factors are required for salivary gland cell death. ${ }^{25,27,30,31}$

We sought to identify groups of proteins that were enriched in dying salivary glands that might suggest the involvement of either a cellular process or a genetic pathway in salivary gland cell death. We used Ingenuity Pathway Analysis to analyze the 6-, 12-, and 13.5-h proteomics data sets, and this approach identified the protein ubiquitination pathway as the top category enriched in dying salivary glands. In two-way comparisons, we identified groups of proteins that increased in detection values between 6 and $13.5 \mathrm{~h}$ APF (Supplementary Table S4). The enrichment of a large number of proteasome components and ubiquitination regulators in dying salivary glands suggested a possible role for the UPS in salivary gland cell death (Table 1). These findings suggest several possibilities; UPS components may be enriched in dying salivary glands because the UPS has an increased function during salivary gland cell death. Alternatively, if the UPS has reduced function during cell death, it is possible that dying salivary gland cells attempt to compensate by increasing the levels of UPS components.

UPS function is reduced in dying salivary gland cells. Previous studies in cell lines suggest that inhibition of proteasome function may be part of a normal cell death program. $^{10,11}$ To monitor UPS function during Drosophila salivary gland cell death, we used transgenic fly lines containing UPS reporter substrates that consist of fusions between GFP and degron sequences that target proteins for degradation by the UPS. ${ }^{32-34}$ During proper UPS function, GFP is targeted to the proteasome for degradation, whereas GFP accumulates during decreased flux through the UPS pathway.

The GFP reporter for flux through the UPS pathway (UASGFP-CL1) was expressed specifically in the salivary glands of experimental animals using the salivary gland-specific driver forkhead-GAL4 (fkh-GAL4), whereas a stable GFP reporter (UAS-Ub-MGFP) was expressed in salivary glands of control animals. To monitor UPS function in salivary gland cells, glands were dissected and examined by fluorescence microscopy to assay for GFP fluorescence at stages before (6h APF), during (12 h APF), and after the onset of salivary gland cell death (13.5h APF). All of the control animals possess fluorescence in salivary gland cells 6,12 , and $13.5 \mathrm{~h}$ APF (Figure 1a, top panels). Salivary glands expressing the UPS flux reporter GFP-CL1 exhibit low fluorescence at 6 and 
Table 1 List of UPS components enriched in dying wild-type Drosophila larval salivary glands

\begin{tabular}{|c|c|c|c|}
\hline \multirow[b]{2}{*}{ Symbol } & \multirow[b]{2}{*}{ Notes } & \multicolumn{2}{|c|}{ Wild-type 6 versus 13.5 h } \\
\hline & & Ratio2 & $P$-value \\
\hline ari-1 & Ubiquitin-protein ligase activity & 1.5 & 0.7884 \\
\hline CG17331 & Proteasome core complex & 1.1 & 0.5807 \\
\hline CG1950 & Ubiquitin thiolesterase activity & 1.5 & 0.4562 \\
\hline CG30382 & Proteasome core complex & 1.2 & 0.5712 \\
\hline CG3473 & Ubiquitin-protein ligase activity & 1.2 & 0.9195 \\
\hline CG7656 & Ubiquitin-protein ligase activity & 1.1 & 0.9309 \\
\hline CG8184 & Ubiquitin-protein ligase activity & Inf & 0.2855 \\
\hline CG9086 & Ubiquitin-protein ligase activity & 5.9 & 0.0651 \\
\hline $\mathrm{crl}$ & Ubiquitin-protein ligase activity & Inf & 0.2855 \\
\hline Cul-2 & Ubiquitin-protein ligase binding & 1.2 & 0.9108 \\
\hline Cul-2 & Ubiquitin-protein ligase binding & 1.2 & 0.9108 \\
\hline Cul-5 & Cullin-RING ubiquitin ligase complex & 1.5 & 0.7994 \\
\hline hyd & Ubiquitin-protein ligase activity & 1.1 & 0.9309 \\
\hline Pros25 & Proteasome core complex & 1.5 & 0.0136 \\
\hline Pros26.4 & Proteasome regulatory particle & 1.1 & 0.5846 \\
\hline Pros 45 & Proteasome regulatory particle, base subcomplex & 1.3 & 0.1907 \\
\hline Prosalpha3T & Endopeptidase activity & 1.2 & 0.9195 \\
\hline Prosalpha5 & Proteasome complex & 1.1 & 0.4613 \\
\hline Prosbeta1 & Proteasome core complex & 1.3 & 0.0064 \\
\hline Prosbeta3 & Proteasome core complex & 1.3 & 0.1033 \\
\hline Prosbeta7 & Proteasome core complex & 1.1 & 0.523 \\
\hline Prp19 & Ubiquitin-protein ligase activity & 7.2 & 0.02349 \\
\hline Rpn1 & Proteasome regulatory particle, base subcomplex & 1.5 & 0.0088 \\
\hline Rpn11 & Proteasome regulatory particle, lid subcomplex & 1.1 & 0.5851 \\
\hline Rpn2 & Proteasome regulatory particle, base subcomplex & 1.4 & 0.011 \\
\hline Rpn5 & Proteasome regulatory particle, lid subcomplex & 1.5 & 0.05447 \\
\hline Rpn7 & Proteasome regulatory particle, lid subcomplex & 1.3 & 0.4598 \\
\hline slmb & Ubiquitin-protein ligase activity & Inf & 0.2855 \\
\hline Tbp-1 & Proteasome regulatory particle & 1.6 & 0.03149 \\
\hline UbcD4 & Ubiquitin-protein ligase activity & Inf & 0.2855 \\
\hline UbcD6 & Ubiquitin-protein ligase activity & 1.2 & 0.8294 \\
\hline Ubc-E2H & Ubiquitin-protein ligase activity & 1.1 & 0.9407 \\
\hline Ufd1 like & Proteasome complex, base subcomplex & 2.4 & 0.4157 \\
\hline
\end{tabular}

Abbreviations: Inf, a numerical value divided by 0 . - Inf, 0 divided by a numerical value; UPS, ubiquitin proteasome system.

Protein names are based on Flybase annotation (http://flybase.org/). The ratio2 values for peptides mapped to the genes listed are indicated (ratio $2=$ average detection value in the 13.5-h sample divided by average detection value in the 6 -h sample). $P$-values were obtained using the Student's $t$-test followed by multiple testing adjustment using the Benjamini-Hochberg method. ${ }^{51}$

$12 \mathrm{~h}$ APF (Figure 1a, bottom panels). However, GFP fluorescence increases dramatically in salivary glands by 13.5 h APF (Figure 1a, bottom panels), suggesting that flux through the UPS pathway is decreased in dying salivary glands, perhaps due to a decrease in UPS function. If UPS function is decreased in salivary gland cells during cell death, we would expect ubiquitinated proteins to accumulate in these cells. We analyzed wild-type salivary gland protein extracts by western blot with ubiquitin antibody and found that ubiquitinated proteins increase in salivary gland extracts between 6 and 13.5 h APF (Figure 1b). These results suggest that the flux through the UPS pathway decreases, and the function of the UPS is reduced, during salivary gland cell death.

Ectopic proteasome impairment leads to early salivary gland condensation and DNA fragmentation that is not suppressed by caspase inhibitor p35 expression. Proteasome impairment leads to caspase activation and cell death in cell lines. ${ }^{18}$ To test whether proteasome impairment leads to early cell death in vivo, we expressed an inducible dominant temperature-sensitive mutant allele of the $\beta 2$ subunit of the $20 \mathrm{~S}$ proteasome (UAS-Dts $7^{2 \mathrm{c}}$ ) ${ }^{35}$ specifically in salivary gland cells, using the salivary gland-specific GAL4 driver fkh-GAL4. To impair proteasome function before the onset of salivary gland cell death, animals expressing Dts $7^{2 \mathrm{C}}$ in salivary gland cells were shifted from permissive to restrictive temperature at puparium formation $(0 \mathrm{~h})$ to $8 \mathrm{~h}$ APF. In wild-type animals, salivary gland cell death commences $12 \mathrm{~h}$ APF. As a normal aspect of salivary gland cell death, we observe salivary gland condensation, or a decrease in salivary gland size, at 13-14 h APF. Salivary gland condensation can be measured as a decrease in salivary gland area in histological sections. We found that salivary glands of control animals lacking the GAL4 driver (UAS-Dts7/+) are intact and have a normal size and morphology at $8 \mathrm{~h}$ APF. In contrast, we observed that salivary glands of experimental animals, in which the proteasome is ectopically impaired specifically in salivary gland cells ( $f k h-G A L 4 /+$ UAS-Dts $7^{2 \mathrm{c}} /+$ ), are smaller in size, or more condensed, than those of control animals (Figure 2a). To quantify salivary gland size, we measured the area of salivary gland material in histological sections and found a significant difference in salivary gland area between control animals and those with ectopic proteasome impairment in salivary gland cells (Figure 2e). These data suggest that in vivo ectopic proteasome impairment 
a

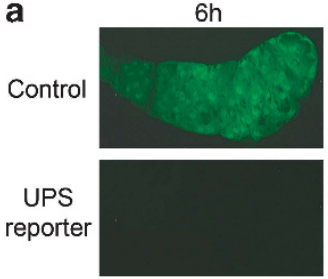

$12 \mathrm{~h}$

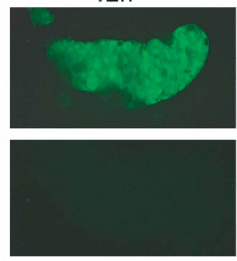

$13.5 \mathrm{~h}$

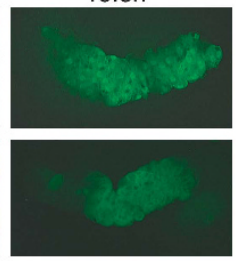

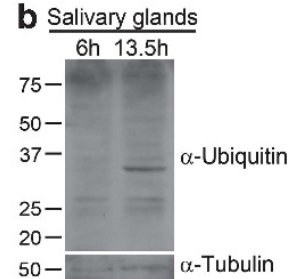

Figure 1 UPS function is reduced during wild-type salivary gland developmental cell death. (a) Control animals ( $f k h-G A L 4 /+$; UAS-Ub-M-GFP/ +) expressing a stable GFP reporter in salivary glands exhibit GFP fluorescence in salivary glands 6,12 , and $13.5 \mathrm{~h}$ AFP, $n=11$. Pupae with salivary gland-specific expression of a GFP reporter for UPS impairment ( $f k h-G A L 4 /+$; UAS-GFP-CL1/ + ) exhibit low GFP fluorescence in salivary glands at 6 and $12 \mathrm{~h}$ AFP, but intense GFP fluorescence in salivary glands 13.5 h AFP, $n=29$. (b) Protein extracts from wild-type salivary glands 6 and 13.5h AFP were analyzed by western blotting with an anti-ubiquitin antibody

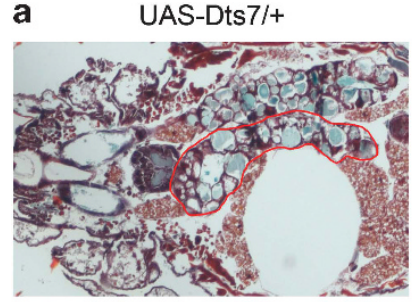

b

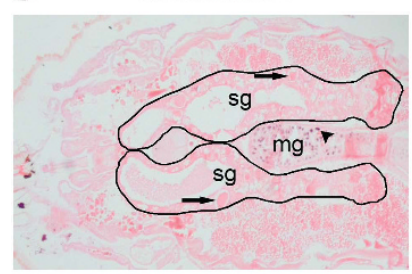

fkh-GAL4; UAS-Dts7

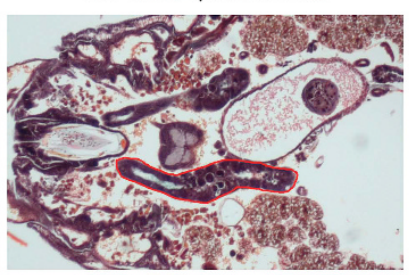

fkh-GAL4-UAS-Dts7
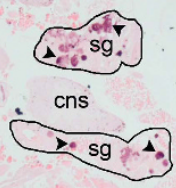

C

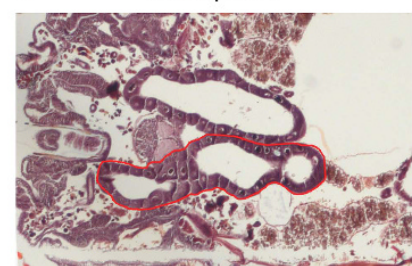

d UAS-p35/+; UAS-Dts7/+



fkh-GAL4; UAS-p35

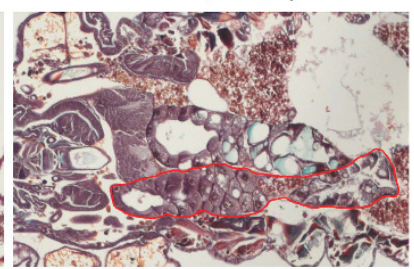

fkh-GAL4; UAS-p35; UAS-Dts7

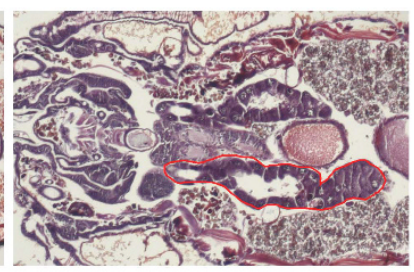

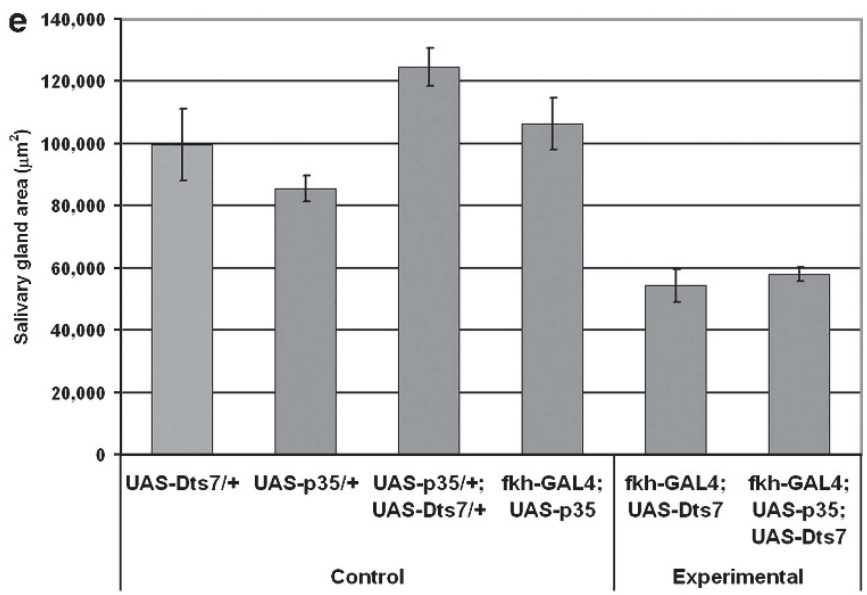

Figure 2 Ectopic proteasome impairment leads to early salivary gland condensation that is not suppressed by caspase inhibitor p35 expression. Pupae were shifted from permissive $\left(25^{\circ} \mathrm{C}\right)$ to restrictive $\left(28^{\circ} \mathrm{C}\right)$ temperature at puparium formation $(0 \mathrm{~h})$, fixed $8 \mathrm{~h} \mathrm{AFP}$, and processed for histology. (a) Control animals lacking the GAL4 driver $\left(+/\right.$ UAS-Dts $\left.7^{2 \mathrm{C}}\right), n=9$, and those with salivary gland-specific expression of Dts $7^{2 \mathrm{C}}$ to genetically impair proteasome function (fkh-GAL4/ $+; \mathrm{UAS}-\mathrm{Dts} 7^{2 \mathrm{C}} /+$ ), $n=13$, were analyzed by histology $8 \mathrm{~h}$ after temperature shift for salivary gland material (outlined in red). Gland area is shown in (e). (b) Control animals $\left(+/\right.$ UAS-Dts $\left.{ }^{2 \mathrm{C}}\right), n=10$, and those with salivary gland-specific expression of Dts $7^{2 \mathrm{C}}$ to impair proteasome function ( $f k h$-GAL4/ + ; UAS-Dts $\left.7^{2 \mathrm{C}} /+\right), n=12$, were in situ TUNEL-labeled to assay for DNA fragmentation. Salivary glands (sg) of control animals lacking the GAL4 driver (+/UAS-Dts $7^{20}$ ) do not possess fragmented DNA, as indicated by light TUNEL-negative salivary gland nuclei (arrows), whereas internal control midgut (mg) nuclei possess fragmented DNA (arrowheads), as indicated by dark TUNEL-positive staining. Salivary glands of experimental animals ( $f k h-G A L 4 /+$; UAS-Dts $7^{2 C} /+$ ) contain fragmented DNA (arrowheads), as indicated by dark TUNEL-positive staining, following UPS impairment. Cns, central nervous system; mg, midgut; Sg, salivary glands. (c) Control animals lacking the GAL4 driver (UAS-p35/ + ), $n=9$, and those expressing p35 in salivary glands ( $f k h-G A L 4 /+;$ UAS-p35/ +), $n=18$, were analyzed for salivary gland material (outlined in red). Gland area is shown in (e). (d) Control animals lacking the GAL4 driver (UAS-p35/ +; UAS-Dts $\left.7^{2 c} /+\right), n=12$, and those expressing p35 in the salivary glands ( $f k h-G A L 4 /+;$ UAS-p35/ + ; and UAS-Dts $7^{2 c /}+$ ), $n=15$, were analyzed for salivary gland material (outlined in red). Gland area is shown in (e). (e) For all slides of genotypes shown in (a, b, and $\mathbf{d}$ ), the largest salivary gland section per slide was outlined for measurement, and salivary gland area was measured using the Zeiss AxiovisionLE software. Red outlines designate the area measured. Error bars represent S.E. 
in salivary gland cells leads to the early onset of salivary condensation.

We next tested whether ectopic proteasome impairment in salivary gland cells leads to early caspase activation in vivo. DNA fragmentation is a caspase-dependent process in salivary glands. ${ }^{26}$ We used the TUNEL assay to detect DNA fragmentation in response to ectopic proteasome impairment. Control animals and those expressing Dts $7^{2 \mathrm{C}}$ in salivary gland cells were shifted from permissive to restrictive temperature at puparium formation $(0 \mathrm{~h})$ to $8 \mathrm{~h}$ APF, and histological sections were TUNEL stained to assay for DNA fragmentation. We found that whereas the nearby nuclei of the larval midgut of control pupae stain positively for TUNEL at this stage, their salivary gland cells do not (Figure $2 \mathrm{~b}$ ). By contrast, salivary gland cells of pupae expressing Dts $7^{2 \mathrm{c}}$ contain fragmented DNA as indicated by dark TUNEL-positive salivary gland nuclei (Figure 2b). These data suggest that ectopic proteasome impairment in salivary gland cells leads to early DNA fragmentation in vivo.

To test whether the early reduction in salivary gland size upon ectopic proteasome impairment is caspase dependent, we co-expressed the caspase inhibitor p35 with $D$ ts $7^{2 \mathrm{c}}$ in salivary glands. We found that salivary glands of pupae expressing p35 alone are not significantly different in size compared with control salivary glands (Figures $2 \mathrm{c}$ and $\mathrm{e}$ ). Surprisingly, however, we found that salivary glands of pupae co-expressing p35 and Dts $7^{2 \mathrm{C}}$ still undergo significant early condensation (Figures $2 \mathrm{~d}$ and $\mathrm{e}$ ). These data suggest that although DNA becomes fragmented following ectopic proteasome impairment in salivary gland cells, the early condensation of salivary glands following proteasome impairment may not be caspase-dependent.

\section{Identification of proteins altered following ectopic} proteasome impairment that are required for salivary gland degradation. Drugs targeting the proteasome are used in anticancer therapies. ${ }^{19,20}$ To better understand cellular responses to altered proteasome function, we conducted a proteomics study of salivary glands following ectopic proteasome impairment. At least three independent biological replicates for each of the control and proteasome impaired salivary gland samples were analyzed using highthroughput shotgun proteomics (Supplementary Table S1). In the proteasome impairment samples (fkh-GAL4/ + ; UASDts $7^{2 \mathrm{c}} /+$ ), an average of 12601 distinct peptides that mapped to an average of 4184 distinct proteins were identified (Supplementary Table S2). In control samples that lack the GAL4 driver ( + /UAS-Dts $7^{2 \mathrm{C}}$ ), an average of 12094 distinct peptides that mapped to an average of 3922 distinct proteins were identified (Supplementary Table S2). Proteins identified in the proteasome impairment and control samples are listed in Supplementary Table S5. An additional $f k h$ GAL4/ + control was collected and analyzed by proteomics to control for proteins that may be enriched in response to GAL4 expression (data not shown).

We sought to identify genes that are required for salivary gland cell degradation. We queried our proteomics data for proteins that were enriched (indicated by positive ratio2 values) in (1) a two-way comparison between the control $(+1$ UAS-Dts $7^{2 \mathrm{C}}$ ) and ectopic proteasome impairment ( fkh-GAL4/ + ;
UAS-Dts $7^{2 \mathrm{c}} /+$ ) data sets and also in (2) a two-way comparison between the 6- and 13.5-h APF wild-type dying salivary gland data sets (Supplementary Table S6). Of these, proteins detected in the proteasome impairment sample with $P$-values $\leq 0.002$ that were also not previously shown to function in salivary gland degradation were considered top candidates for genetic analyses. From this list of candidate proteins, eight were identified with available transgenic RNAi lines (CG11880, CG3074, CG9336, CG9917, CG9977, Dbi, Ect3, and Trol), and these were all screened for defects in salivary gland cell degradation.

To knockdown genes specifically in salivary gland cells, transgenic fly lines containing UAS-RNAi constructs were crossed to those expressing fkh-GAL4. Progeny expressing RNAi in salivary glands were staged to $24 \mathrm{~h}$ APF; a stage that is $8 \mathrm{~h}$ after salivary gland material is cleared in wild-type Drosophila. Of the eight genes tested, two had defects in salivary gland degradation. We found that compared with control animals, those expressing RNAi against the gene encoding Trol possessed persistent salivary gland fragments (Figures $3 a$ and b). Similarly, we found that animals expressing RNAi in salivary glands against CG11880, a gene of unknown function, were deficient in salivary gland degradation compared with controls (Figures $3 c$ and d). All other morphological and developmental events occurred normally in these animals, including the timing and morphology of future adult head eversion. Therefore, the inhibition of salivary gland degradation upon either trol or CG11880 expression in salivary glands was not due to a developmental delay. Trol and CG11880 were identified following proteasome inhibition, which follows activation of caspases in salivary glands. As proteasome inhibition can lead to activation of autophagy, ${ }^{34}$ we tested if these genes are required for autophagy in dying salivary glands. To our surprise, knockdown of neither trol nor CG11880 had any impact on autophagy (data not shown). To our knowledge, this is the first demonstration that trol and CG1180 are required for salivary gland cell degradation.

The cop9 signalsome (CSN) functions in salivary gland degradation. We queried our proteomics data for proteins that are part of a pathway or cellular process and were enriched both in response to ectopic proteasome impairment and during wild-type salivary gland cell death. Interestingly, the CSN network was identified as being enriched by Ingenuity Pathway Analysis. A number of CSN subunits were detected in both wild-type dying salivary glands and in salivary glands with ectopic proteasome impairment (Table 2). Although the $P$-values for the detection of each individual component were relatively high and therefore did not engender high confidence in the detection of any single subunit, the detection of 7 out of 8 known CSN subunits, including $1 \mathrm{~b}, 3,4,5,6,7$, and 8 , suggested a possible role for the CSN in salivary gland death (Table 2).

To test the function of the CSN in salivary gland degradation, we expressed RNAi against the gene encoding CSN subunit 6 (CSN6) in salivary glands. Previous studies have shown that the loss or knockdown of one CSN subunit leads to the destabilization and loss of the entire complex. ${ }^{36-38} \mathrm{We}$ found that whereas $10 \%$ of control animals contained 

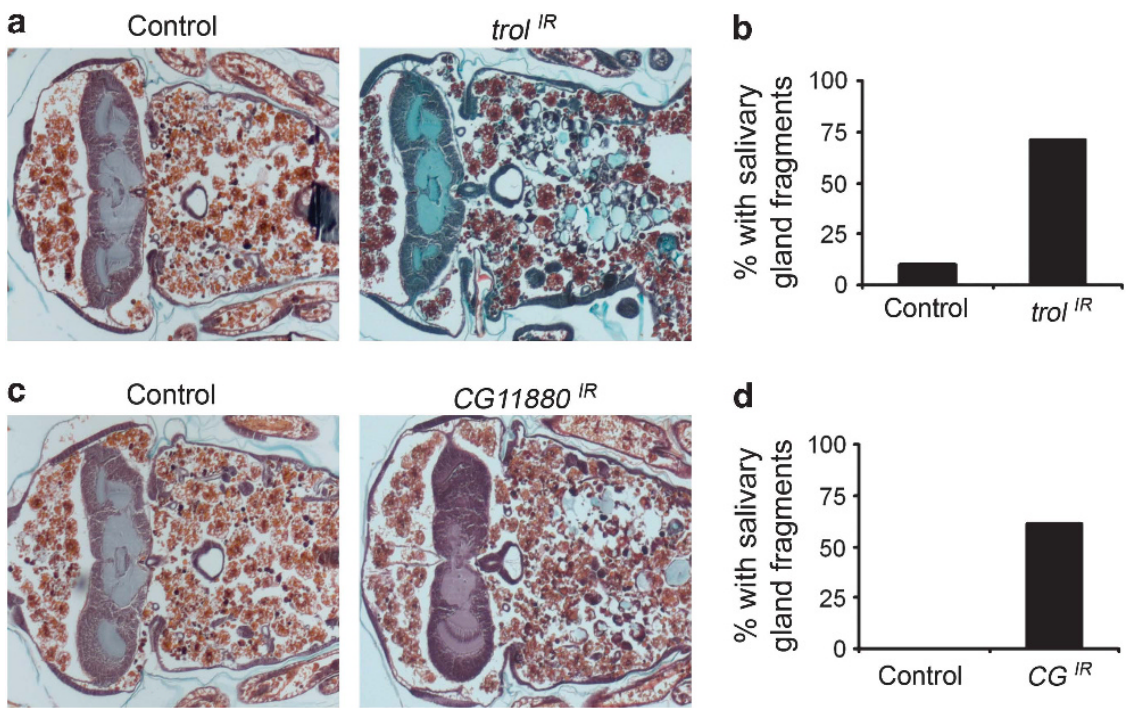

Figure 3 Trol and CG11880 are required for salivary gland cell degradation. (a) Control animals lacking the GAL4 driver $\left(+/ \mathrm{W} ;+/\right.$ UAS-trol $\left.{ }^{I R}\right), n=10$, and those with salivary gland-specific knockdown of trol (fkh-GAL4/w; UAS-trol ${ }^{I R} /+$ ), $n=17$, were analyzed by histology for the presence of salivary glands (red circles) $24 \mathrm{~h}$ AFP. (b) Quantification of data from (a) where $10 \%$ of control animals and $71 \%$ of experimental animals possess persistent salivary gland fragments. (c) Control animals lacking the

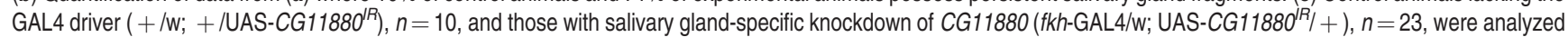
by histology for the presence of salivary glands (red circles) $24 \mathrm{~h} \mathrm{AFP.} \mathrm{(d)} \mathrm{Quantification} \mathrm{of} \mathrm{data} \mathrm{from} \mathrm{(c)} \mathrm{where} 0 \%$ of control animals and $61 \%$ of experimental animals possess persistent salivary gland fragments

Table 2 CSN subunits detected in Drosophila larval salivary glands by proteomics

\begin{tabular}{|c|c|c|c|c|c|}
\hline \multirow[b]{2}{*}{ Symbol } & \multirow[b]{2}{*}{ Notes } & \multicolumn{2}{|c|}{ Proteasome impairment } & \multicolumn{2}{|c|}{ Wild-type 6 versus $13.5 \mathrm{~h}$} \\
\hline & & Ratio2 & $P$-value & Ratio2 & $P$-value \\
\hline CSN1b & Signalosome complex subunit $1 \mathrm{~b}$ & -1.2 & 0.7530 & Inf & 0.2855 \\
\hline CSN3 & Signalosome complex subunit 3 & -1.2 & 0.9047 & 2.0 & 0.4593 \\
\hline CSN4 & Signalosome complex subunit 4 & 1.1 & 0.8339 & -1.6 & 0.6866 \\
\hline CSN5 & Signalosome complex subunit 5 & 1.6 & 0.2789 & 1.8 & 0.1618 \\
\hline CSN6 & Signalosome complex subunit 6 & 1.8 & 0.0716 & -2.6 & 0.4925 \\
\hline CSN7 & Signalosome complex subunit 7 & 1.5 & 0.2554 & 4.6 & 0.2600 \\
\hline CSN8 & Signalosome complex subunit 8 & -2.0 & 0.1428 & ND & ND \\
\hline
\end{tabular}

Abbreviations: CSN, cop9 signalsome; CSN6, cop9 signalsome 6; Inf, a numerical value divided by 0; NA, not applicable; ND, not detected.

Protein names are based on Flybase annotation (http://flybase.org/). The ratio2 values for peptides mapped to the genes listed are indicated (ratio2 $=$ average detection value experimental sample divided by average detection value control sample). $P$-values were obtained using the Student's $t$-test followed by multiple testing adjustment using the Benjamini-Hochberg method. ${ }^{51}$

persistent salivary gland fragments, $100 \%$ of those expressing csn6-IR in salivary glands contained persistent salivary gland fragments $24 \mathrm{~h}$ APF (Figures $4 \mathrm{a}$ and b). We identified csn6 as being enriched after proteasome inhibition. We tested if csn6 is required for autophagy in dying salivary glands as altered proteasome function can lead to activation of autophagy in neurons, ${ }^{34}$ however, knockdown of $\operatorname{csn} 6$ did not influence autophagy (data not shown).

\section{Discussion}

Autophagic programmed cell death appears to be conserved between insects and mammals, ${ }^{25,39}$ yet the mechanisms that contribute to this type of cell death are not well understood. We used a shotgun proteomic approach to identify proteins enriched during autophagic programmed cell death in purified dying Drosophila larval salivary glands. We found that a number of UPS components are enriched in dying salivary glands, suggesting a role for the UPS in salivary gland cell death. Significantly, UPS components are enriched in proteomic studies of apoptotic cells. ${ }^{40}$ We tested the function of the UPS in salivary glands and found that flux through the UPS pathway is decreased during cell death in wild-type salivary glands.

Although the mechanism leading to decreased UPS function during salivary gland cell death remains unknown, it could be that UPS function is reduced by system overload, but other possibilities exist. It is interesting that a large number of UPS components are enriched in dying salivary glands at the time when UPS function is decreased, suggesting a possible compensatory upregulation to deal with a reduction in the function of this important catabolic process. We find that ectopic proteasome impairment leads to the onset of early salivary gland condensation and DNA fragmentation in vivo. Unexpectedly, however, we find that caspase inhibitor p35 expression during proteasome impairment does 

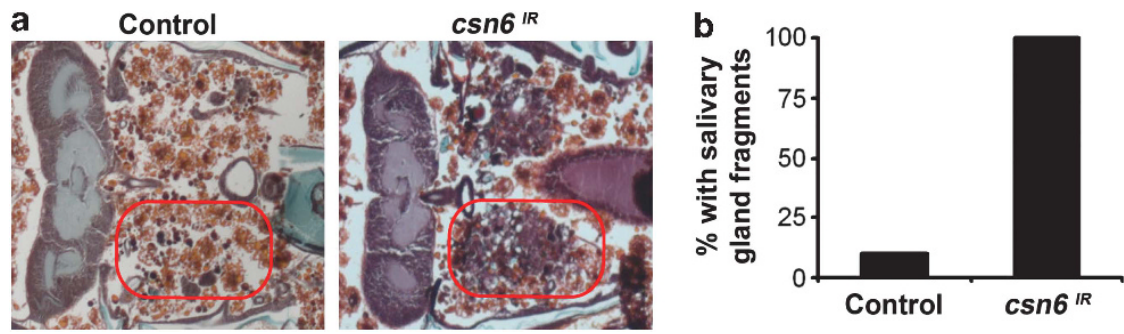

Figure 4 RNAi knockdown of CSN6 prevents salivary gland degradation. (a) Control animals lacking the GAL4 driver $\left(+/\right.$ w; $+/$ UAS-cSn $\left.6^{I R}\right), n=10$, and those with salivary gland-specific knockdown of $\operatorname{csn} 6$ ( $f k h-G A L 4 / w$; UAS- $\left.\operatorname{csn} 6^{(R /} /+\right), n=22$, were analyzed by histology for the presence of salivary glands (red circles) $24 \mathrm{~h}$ AFP. $c s n 6^{R}=$ VDRC TID no. 22308 on II. (b) Quantification of data from (a) where $10 \%$ of control animals and $100 \%$ of experimental animals possess persistent salivary gland fragments

not suppress ectopic salivary gland condensation. This suggests that proteasome impairment-induced gland condensation may require the activity of caspase-independent processes.

We queried our proteomics data to identify factors present in salivary glands upon proteasome impairment. To identify physiologically relevant factors that might function in salivary gland cell degradation, we compared factors identified following ectopic proteasome impairment in salivary glands to those identified during wild-type salivary gland cell death. The simple presence of proteins in dying salivary glands is not evidence that these proteins function in cell death and degradation. Therefore, we took a genetic approach to screen top candidates for defects in salivary gland degradation by RNAi knockdown. Here, we show that the knockdown of trol and CG11880, a gene of unknown function, prevent proper salivary gland degradation. The gene trol has been shown to function in controlling the onset of stem cell proliferation in the developing Drosophila nervous system by modulating fibroblast growth factor branchless and Hedgehog signaling, and mutations in trol also affect Dpp and Wingless signaling. ${ }^{41}$ However, neither CG11880 nor trol has previously been shown to function in salivary gland cell degradation.

To identify additional genes that might function in autophagic cell death, we searched for proteins that are members of a pathway or cellular component and present during wild-type cell death and in response to proteasome impairment in salivary glands. We identified 7 out of 8 known CSN components by proteomics and demonstrated that RNAi knockdown of CSN subunit csn6 prevents salivary gland degradation. The CSN is a conserved multi-subunit protein complex that functions in the ubiquitin/proteasome pathway. ${ }^{42}$ First discovered in plants, the CSN is essential for Drosophila development and functions in a variety of essential cellular processes, including cell cycle control, the DNA-damage response, and circadian rhythm. ${ }^{42}$ Composed of eight subunits, CSN1-CSN8, the CSN contains Nedd8 isopeptidase activity, and functions to remove the ubiquitin-like Nedd8 from proteins (deneddylate). ${ }^{42}$

Our finding that csn6 is required for salivary gland degradation demonstrates a role for the CSN in autophagic cell death, and suggests that proper control of protein neddylation and deneddylation cycles is important to regulate salivary gland degradation. Several studies have demonstrated a role for the CSN in regulating cell death. Csn5 knockout mice exhibit elevated apoptosis. ${ }^{43,44}$ In addition, several cullin-RING ligase components negatively regulate Caenorhabditis elegans p53 (CEP-1)-dependent germ cell apoptosis in response to DNA damage. ${ }^{45}$ Cullin-RING ligase complexes may also function in salivary gland cell death, a subject for future investigation.

Our proteomics analyses led to the discovery of several genes required for proper salivary gland cell degradation. These findings exhibit the power of a high-throughput proteomics approach to identify factors that function in biological processes. Our proteomics data will serve as a foundation for future genetic studies of autophagic programmed cell death and in vivo cellular responses to proteasome inhibition by providing a description of proteins enriched during wild-type cell death as well as in response to ectopic proteasome impairment.

\section{Materials and Methods}

Drosophila strains. The Canton-S strain was used as wild-type. For ectopic proteasome impairment studies, UAS-Dts $7^{2 \mathrm{C}}$ on III was used. ${ }^{35}$ To monitor proteasome impairment, UAS-M-GFP was used as the positive control, and UAS-GFP-CL1 was used as the experimental stock. ${ }^{32-34}$ For ectopic p35 expression, UAS-p35 was used. ${ }^{46}$ The following Vienna Drosophila RNAi Center (VDRC, Vienna, Austria) RNAi stocks were used: $C G 11880^{R}$ VDRC Transpformant ID (TID) 22867 on II, trol/R VDRC TID no. 245498 on II.

Proteomics analysis. To generate the proteomics data sets, at least three independent biological samples for each condition were collected. For each independent sample, 80 pairs of salivary glands were dissected per $100 \mu \mathrm{l} 8 \mathrm{M}$ urea/40 mM Tris- $\mathrm{HCl}, \mathrm{pH} 8$. At least two runs of each biological sample were analyzed, using $10 \mu \mathrm{g}$ of sample per run, by high-throughput MS-based proteomics. ${ }^{47}$ Proteins were fully trypsinized, and peptides were separated by two-dimensional chromatography; first into 15 fractions by capillary isoelectric focusing, and further resolved by capillary reverse-phase liquid chromatography (RPLC) (nano-RPLC). Two runs of each protein sample were carried out. Fractions were analyzed by ESI-tandem-MS using a Thermo Finnigan LTQ linear ion trap mass spectrometer (San Jose, CA, USA). Following MS analysis, peaklists were generated using extract_msn, and searches to identify MS/MS peptide spectra were carried out using the Open Mass Sepctrometry Search Algorithm $^{48}$ (NCBI). For each data set, protein and peptide hits were generated assuming a false positive rate of $5 \%$, and proteins present in the samples were identified based upon peptide sequences detected. Comparison of proteomics data sets: Files containing flybase gene identifiers (FBGNs), flybase gene symbols, and RefSeq protein accession numbers were batch downloaded from http://www.flybase.org. Microsoft Access (Seattle, WA, USA) was then used to merge proteomics data files with that containing FBGN, flybase gene symbols, and RefSeq accessions. Using Microsoft Access, separate proteomics data sets containing ratio2 values were then merged based upon their common FBGN numbers. This allowed the comparison of ratio2 values and $P$-values between proteins identified in more than one proteomics data set.

Histology. Histology was performed as previously described. ${ }^{49}$ 
Protein extracts and western blotting. Protein extraction and western blotting were performed as previously described. ${ }^{50}$ Primary antibodies used were rabbit anti-ubiquitin (1:1000), and mouse anti-beta-tubulin $(1: 50 ; z)$.

Microscopy. Imaging was performed on a Zeiss (New York, NY, USA) Axiophot II microscope. To quantify salivary gland size, the area of the largest salivary gland section per pupa was measured using the Zeiss AxioVisionLE software.

\section{Conflict of Interest}

The authors declare no conflict of interest.

Acknowledgements. We thank P Meier, the Bloomington Stock Center, and the VDRC for Drosophila strains. We thank K Simin for assistance with proteomics data analysis. This work was supported by the NIH grant GM079431 to EHB. EHB is an Ellison Medical Foundation Scholar and a member of the UMass DERC (DK32520).

1. Lockshin RA, Williams CM. Programmed cell death-I. Cytology of degeneration in the intersegmental muscles of the pernyi silkmoth. J Insect Physiol 1965; 11: 123-133.

2. Danial NN, Korsmeyer SJ. Cell death: critical control points. Cell 2004; 116: 205-219.

3. Thompson CB. Apoptosis in the pathogenesis and treatment of disease. Science 1995; 267: $1456-1462$.

4. Clarke PGH. Developmental cell death: morphological diversity and multiple mechanisms. Anat Embryol 1990; 181: 195-213.

5. Schweichel J-U, Merker H-J. The morphology of various types of cell death in prenatal tissues. Teratology 1973; 7: 253-266.

6. Ciechanover A. Proteolysis: from the lysosome to ubiquitin and the proteasome. Nat Rev Mol Cell Biol 2005; 6: 79-87.

7. Schwartz AL, Ciechanover A. The ubiquitin-proteasome pathway and pathogenesis of human diseases. Annu Rev Med 1999; 50: 57-74.

8. Wang J, Maldonado MA. The ubiquitin-proteasome system and its role in inflammatory and autoimmune diseases. Cell Mol Immunol 2006; 3: 255-261.

9. Drexler HC. Programmed cell death and the proteasome. Apoptosis 1998; 3: 1-7.

10. Sun XM, Butterworth M, MacFarlane M, Dubiel W, Ciechanover A, Cohen GM. Caspase activation inhibits proteasome function during apoptosis. Mol Cell 2004; 14: 81-93.

11. Adrain C, Creagh EM, Cullen SP, Martin SJ. Caspase-dependent inactivation of proteasome function during programmed cell death in Drosophila and man. J Biol Chem 2004; 279: 36923-36930

12. Schwartz LM, Myer A, Kosz L, Engelstein M, Maier C. Activation of polyubiquitin gene expression during developmentally programmed cell death. Neuron 1990; 5 : 411-419.

13. Jones ME, Haire MF, Kloetzel PM, Mykles DL, Schwartz LM. Changes in the structure and function of the multicatalytic proteinase (proteasome) during programmed cell death in the intersegmental muscles of the hawkmoth, Manduca sexta. Dev Biol 1995; 169: 436-447.

14. Dawson SP, Arnold JE, Mayer NJ, Reynolds SE, Billett MA, Gordon C et al. Developmental changes of the $26 \mathrm{~S}$ proteasome in abdominal intersegmental muscles of Manduca sexta during programmed cell death. J Biol Chem 1995; 270: 1850-1858.

15. Takayanagi K, Dawson S, Reynolds SE, Mayer RJ. Specific developmental changes in the regulatory subunits of the $26 \mathrm{~S}$ proteasome in intersegmental muscles preceding eclosion in Manduca sexta. Biochem Biophys Res Commun 1996; 228: 517-523.

16. Shinohara K, Tomioka M, Nakano H, Tone S, Ito H, Kawashima S. Apoptosis induction resulting from proteasome inhibition. Biochem J 1996; 317(Part 2): 385-388.

17. Drexler HC. Activation of the cell death program by inhibition of proteasome function. Proc Natl Acad Sci USA 1997; 94: 855-860.

18. Henderson CJ, Aleo E, Fontanini A, Maestro R, Paroni G, Brancolini C. Caspase activation and apoptosis in response to proteasome inhibitors. Cell Death Differ 2005; 12: 1240-1254.

19. Hoeller D, Dikic I. Targeting the ubiquitin system in cancer therapy. Nature 2009; 458: 438-444.

20. Tobinai K. Proteasome inhibitor, bortezomib, for myeloma and lymphoma. Int J Clin Oncol 2007; 12: 318-326.

21. Adams J. The development of proteasome inhibitors as anticancer drugs. Cancer Cell 2004; 5: 417-421

22. Ling $\mathrm{YH}$, Liebes L, Jiang JD, Holland JF, Elliott PJ, Adams J et al. Mechanisms of proteasome inhibitor PS-341-induced G(2)-M-phase arrest and apoptosis in human nonsmall cell lung cancer cell lines. Clin Cancer Res 2003; 9: 1145-1154.

23. Meister S, Schubert U, Neubert K, Herrmann K, Burger R, Gramatzki M et al. Extensive immunoglobulin production sensitizes myeloma cells for proteasome inhibition. Cancer Res 2007; 67: 1783-1792.
24. Gu H, Chen X, Gao G, Dong $H$. Caspase-2 functions upstream of mitochondria in endoplasmic reticulum stress-induced apoptosis by bortezomib in human myeloma cells. Mol Cancer Ther 2008; 7: 2298-2307.

25. Berry DL, Baehrecke EH. Growth arrest and autophagy are required for salivary gland cell degradation in Drosophila. Cell 2007; 131: 1137-1148.

26. Lee C-Y, Baehrecke EH. Steroid regulation of autophagic programmed cell death during development. Development 2001; 128: 1443-1455.

27. Martin DN, Baehrecke EH. Caspases function in autophagic cell death in Drosophila. Development 2004; 131: 275-284.

28. Lee C-Y, Clough EA, Yellon P, Teslovich TM, Stephan DA, Baehrecke EH. Genome-wide analyses of steroid- and radiation-triggered programmed cell death in Drosophila. Curr Biol 2003; 13: 350-357.

29. Martin DN, Balgley B, Dutta S, Chen J, Rudnick P, Cranford J et al. Proteomic analysis of steroid-triggered autophagic programmed cell death during Drosophila development. Cell Death Differ 2007; 14: 916-923.

30. Lee CY, Wendel DP, Reid P, Lam G, Thummel CS, Baehrecke EH. E93 directs steroidtriggered programmed cell death in Drosophila. Mol Cell 2000; 6: 433-443.

31. Yao T-P, Forman BM, Jiang Z, Cherbas L, Chen JD, McKeown $M$ et al. Functional ecdysone receptor is the product of $\mathrm{EcR}$ and ultraspiracle genes. Nature 1993; 366: 476-479.

32. Dantuma NP, Lindsten K, Glas R, Jellne M, Masucci MG. Short-lived green fluorescent proteins for quantifying ubiquitin/proteasome-dependent proteolysis in living cells. Nat Biotechnol 2000; 18: 538-543.

33. Bence NF, Bennett EJ, Kopito RR. Application and analysis of the GFPu family of ubiquitinproteasome system reporters. Methods Enzymol 2005; 399: 481-490.

34. Pandey UB, Nie Z, Batlevi Y, McCray BA, Ritson GP, Nedelsky NB et al. HDAC6 rescues neurodegeneration and provides an essential link between autophagy and the UPS. Nature 2007; 447: 859-863.

35. Belote JM, Fortier E. Targeted expression of dominant negative proteasome mutants in Drosophila melanogaster. Genesis 2002; 34: 80-82.

36. Busch S, Schwier EU, Nahlik K, Bayram O, Helmstaedt K, Draht OW et al. An eight-subunit COP9 signalosome with an intact JAMM motif is required for fungal fruit body formation. Proc Natl Acad Sci USA 2007; 104: 8089-8094.

37. Serino G, Deng XW. The COP9 signalosome: regulating plant development through the control of proteolysis. Annu Rev Plant Biol 2003; 54: 165-182.

38. Su H, Huang $\mathrm{W}$, Wang $\mathrm{X}$. The COP9 signalosome negatively regulates proteasome proteolytic function and is essential to transcription. Int J Biochem Cell Biol 2009; 41: 615-624.

39. Elgendy M, Sheridan C, Brumatti G, Martin SJ. Oncogenic Ras-induced expression of Noxa and Beclin-1 promotes autophagic cell death and limits clonogenic survival. Mol Cell 2011; 42: 23-35

40. Arntzen MØ, Thiede B. ApoptoProteomics an integrated database for analysis of proteomics data obtained from apoptotic cells. Mol Cell Proteomics 2012; 11, M111.010447.

41. Lindner JR, Hillman PR, Barrett AL, Jackson MC, Perry TL, Park Y et al. The Drosophila Perlecan gene trol regulates multiple signaling pathways in different developmental contexts. BMC Dev Biol 2007; 7: 121.

42. Wei N, Serino G, Deng XW. The COP9 signalosome: more than a protease. Trends Biochem Sci 2008; 33: 592-600.

43. Tomoda K, Yoneda-Kato N, Fukumoto A, Yamanaka S, Kato JY. Multiple functions of Jab1 are required for early embryonic development and growth potential in mice. J Biol Chem 2004; 279: 43013-43018.

44. Panattoni M, Sanvito F, Basso V, Doglioni C, Casorati G, Montini E et al. Targeted inactivation of the COP9 signalosome impairs multiple stages of $T$ cell development. J Exp Med 2008; 205: 465-477

45. Gao MX, Liao EH, Yu B, Wang Y, Zhen M, Derry WB. The SCF FSN-1 ubiquitin ligase controls germline apoptosis through CEP-1/p53 in C. elegans. Cell Death Differ 2008; 15: 1054-1062.

46. Hay BA, Wolff T, Rubin GM. Expression of baculovirus P35 prevents cell death in Drosophila. Development 1994; 120: 2121-2129.

47. Chen J, Balgley BM, DeVoe DL, Lee CS. Capillary isoelectric focusing-based multidimensional concentration/separation platform for proteome analysis. Anal Chem 2003; 75: 3145-3152.

48. Geer LY, Markey SP, Kowalak JA, Wagner L, Xu M, Maynard DM et al. Open mass spectrometry search algorithm. J Proteome Res 2004; 3: 958-964.

49. Muro I, Berry DL, Huh JR, Chen CH, Huang H, Yoo SJ et al. The Drosophila caspase Ice is important for many apoptotic cell deaths and for spermatid individualization, a nonapoptotic process. Development 2006; 133: 3305-3315

50. Dutta S, Baehrecke EH. Warts is required for PI3K-regulated growth arrest, autophagy, and autophagic cell death in Drosophila. Curr Biol 2008; 18: 1466-1475.

51. Hochberg Y, Benjamini Y. More powerful procedures for multiple significance testing. Stat Med 1990; 9: 811-818. 